\title{
CRISPR/Cas9 system - A revolution in gene editing
}

\author{
Nikolai Adamov ${ }^{1}$, Volodia Georgiev ${ }^{2}$ and Teodora Daneva ${ }^{3 *}$ \\ ${ }^{1}$ Faculty of Chemistry and Pharmacy, Sofia University, Sofia, Bulgaria \\ ${ }^{2}$ Manhattanville College, Purchase, NY, USA \\ ${ }^{3}$ Institute of Biology and Immunology of Reproduction, Bulgarian Academy of Sciences, Sofia, Bulgaria
}

\begin{abstract}
CRISPR/Cas9 technology, is a fundamental and very important discovery for modern biology which we consider simply as a way of editing the genome. It lies in the fact that a huge number of bacteria carry in their genome an effective system of adaptive immunity against potential viral invasion. The basis of this system is special genome regions - short palindromic cluster repeats or CRISPR (Clustered Regularly Interspaced Short Palindromic Repeats).

Ultimately, CRISPR/Cas9 technology can change the standpoint of humanity to many different hereditary diseases. If earlier they were either completely incurable, or they allowed palliative, symptomatic treatment, now it is possible to treat them by manipulating individual genes, that is, to eliminate the very cause of the respective disease. Simultaneously with the advent of genome editing technology, the possibility of its "improvement" appears, in a variety of ways. So far fairly simple (from the point of view of the inheritance mechanism) diseases, and not only mutated genes can be potentially targets for editing, as well as numerous genes associated with an increased risk to human health. Much additional work remains to be done in the areas nucleotide manipulation and replacement before the full therapeutic potential of these approaches can be realized. Of equal importance, all moral and ethical issues related to gene editing therapies must be taken in account before any practical approach is applied.
\end{abstract}

\section{Introduction}

Different technologies have been developed to manipulate DNA, but until recently they were costly, labor-intensive and timeconsuming. Discovery of the Clustered Regularly-Interspaced Short Palindromic Repeats (CRISPR), the mechanism of the CRISPR-based prokaryotic adaptive immune system (CRISPR-associated system, Cas), and its transformation into a potent DNA editing tool has changed completely the field of gene manipulation. CRISPR/Cas9 genome editing was derived from the naturally occurring defense mechanism used by bacteria to shield themselves from infection by bacteriophages and mobile genetic elements. In nature, CRISPR functions as a bacterial adaptive immune system, releasing RNAs that are complementary to intruding DNA - marking them for destruction. After the initial intensive period of data accumulation, CRISPR was soon adapted for a vast range of applications - creating complex animal models of human-inherited diseases and cancers; performing genomewide screens in human cells to define the genes responsive for specific biological processes; regulating the expression of specific genes; used to genetically modifying plants. This technology has opened countless opportunities for easy, inexpensive, and quick editing of human genes. It literally has the potential to change the human medicine and health. Why did this sudden revolution happen and how does it work?

\section{History and origin}

Humans have been engineering life for thousands of years. Since ancient times, mankind has changed the course of evolution through an artificial selection cultivating the scars of plants and animals obtained through random mutations that are most useful and meaningful. Through selective breeding, we strengthened useful traits in plants and animals. This process, however, takes a long time. We become very good at this, but never fully understood how it worked until we discover the DNA. In the $20^{\text {th }}$ century, the structure of DNA was discovered, and many opportunities for exploration were discovered. In the 1970s, attempts were made to accelerate these mutations by treating plant cultures with various mutagenic factors as radiation or chemicals and screening the useful ones. In the 1970's scientists inserted DNA snippets into bacteria, plants, and animals to modify them for research, medicine, agriculture, and even for fun. This marks the beginning of gene engineering. Today we produce many substances by gene engineering as life-saving clotting factors, hormones as insulin and growth hormone, growth factors and others. All things we had to harvest from the organs of animals before that.

During their evolution bacteria have developed a set of barriers to protect themselves against invaders such as phage and plasmid nucleic acids. Different prokaryotic defense systems exist and at least two of them directly target the incoming DNA: Restriction-Modification (RM) and CRISPR-Cas systems. Both systems are compatible and act together to increase the bacterial resistance to phages by cleaving their respective target sites and to decrease phage contaminations.

Specific sequence of recurrent genes in E. Coli was identified, showing five highly homologous sequences of 29 nucleotides, arranged as direct repeats with 32 nucleotides as spacing [1].

Haloferax Mediterranei is an archaebacterial organism with extreme salt tolerance and the high salt concentration affect the way in which restriction enzymes cut the microbe's genome. In the DNA fragments examined, multiple copies of a near-perfect, roughly

*Correspondence to: Teodora Daneva, $\mathrm{PhD}$, Institute of Biology and Immunology of Reproduction, Bulgarian Academy of Sciences, Sofia, Bulgaria, E-mail: danevadoki@abv.bg

Received: October 11, 2018; Accepted: October 22, 2018; Published: October 25,2018 
palindromic, repeated sequence of 30 bases were found, separated by spacers of roughly 36 bases-that did not resemble any family of repeats known in microbes [2].

The name CRISPR - Clustered Regularly Interspaced Short Palindromic Repeats was proposed by Jansen, et al. [3]. In 2005 it was found that the repeats in the sequence were genomes from bacteriophages or other extrachromosomal origin [4]. In 2007 was reported that CRISPR provides resistance against viruses in prokaryotes $[5,6]$.

The CRISPR-Cas system is a bacterial "immune system" against bacteriophages. It is a sequence of the bacterial genome that consists of repeatable short palindromic DNA regions of 30-40 nucleotide pairs in length. They are separated by sections of DNA called "spacers". These regions are different and found to match perfectly with the bacteriophage DNA that the bacterium has already encountered. Associated with the CRISPR sequence are regions of the DNACas genes (CRISPR associated sequences) that encode CAS protein information. They are mainly helicases and nucleases. Because of this CRISPR is called the "immune system of bacteria" [7,8]. In 2009 Hale, et al. published evidence that the CRISPR-Cas system protects prokaryotes from viruses and other potential genome invaders via an unique RNA silencing system that functions by homology-dependent cleavage of invader RNAs [9]. In 2010 Maraffini and Sontheimer have defined the mechanism of CRISPR self/non-self recognition via small CRISPR RNAs (crRNAs) that contain a full spacer flanked by partial repeat sequences [10-12].

The CRISPR/Cas "immune system" is remarkably adapted to cleave invading DNA rapidly and has the potential to generate safer microbial strains [13].

\section{How CRISPR works}

When the bacteriophage infects bacteria and injects the viral DNA into the bacterial cell, the viral genome will force the cell to produce the viral proteins and the viral enzymes and then it will change the whole cell machinery of bacterium. Thankfully to the CRISPR system bacterial cell can prevent this from happening second time. It is a kind of memory to prevent the same bacteriophage from infecting the cell another time. The CRISPR system is a three step mechanism. The first is a Spacer Acquisition, the second is a crRNA Processing, and the third is an Interference and target degradation. Than we have to know that the CRISPR system has three types: type I, type II, and type III depending on bacterial cell. When the bacteriophage infects for the first time bacterial cell, the bacterial cell chop up the virus genome, and takes a piece of it and inserts this piece into the spacer DNA in bacterial genome. Each time when the virus infects the bacterial cell the cell takes a piece of it and inserts it into the spacer DNA. The Cas enzymes are a family of enzymes that are implicated in the CRISPR process. Most of the Cas enzymes are nucleases or helicases.

In the Spacer Acquisition they are two main players - Cas1 and Cas2. Both of them are dimers that form a complex together in order to form a Spacer Acquisition. Cas1 might have a nuclease and integrase activity. They can cut the viral genome and integrate it in-between DNA. Cas2 are endoribonucleases and can mainly cut the RNA (some bacteriophages have RNA genome).

They are three different types of crRNA Processing. In the type I the CRISPR repeats form loops and then the messenger mRNA will be cut by Cas6e or Cas6f. The mRNA is going to become chopped up on small pieces of RNA and each piece contains a loop and the piece of the viral genome. These small pieces are the crRNA. In type II processing are another player called tracrRNA (trans activating crispr RNA). These are RNA pieces that bind to the crRNA on the mRNA. Then the mRNA will be chopped up by Cas 9 and RNase III on pieces consisting of piece of viral genome, crRNA, and trRNA. In type III Cas6 homolog is going to chop up the mRNA directly on a piece of viral genome and crRNA.

The $3^{\text {rd }}$ step Interference is also different between the three types of CRISPR system. But in general the Cas protein and crRNA interfere together and the crRNA will be integrated into the Cas protein to form a complex containing the Cas protein with the piece of crRNA. The difference is between the three types. The type I when the virus infects second time bacteria the crRNA will bind to the complementary strand of the viral genome. This binding will activate a cascade of Cas enzymes to chop up and degrade the viral genome. In type II the main player is Cas9. After the viral DNA and crRNA bind together Cas9 itself performs double strand break (DSB) in the viral genome. The Cas9 has two domains called $\mathrm{HNH}$ and RuvC (RNase H-like endonuclease domains). The Cas 9 can use these endonuclease domains to perform the DSB. In type I and type II Interference PAM (Proto Spacer Adjacent Motif) is a very important player. When the virus infects bacteria, a bacterium takes the piece of the viral genome which is adjacent to the PAM sequence. The bacterial cell can recognize the PAM sequence and then it takes the adjacent sequence in order to add it into the spacer DNA and then in order to form the crRNA and the CRISPR complex from it. Why the PAM is important? Because the not only the RNA but also Cas enzyme can recognize the PAM sequence. PAM increases the specificity of recognition. PAM is very important in type I and type II but not in type III Interference. In type III there also is a Cas enzyme, crRNA, but not PAM and there is also cascade of enzymes as in type I which destroy the viral genome. (Figure 1)

In $2011 \mathrm{Babu}$, et al. report that, in addition to antiviral immunity, at least some components of the CRISPR-Cas system have a function in DNA repair [14].

In 2011 was published that active CRISPR/Cas systems can be transferred across distant genera and provide heterologous interference against invasive nucleic acids [15]. In 2012 Gasiunas, et al. demonstrate that the Cas9-crRNA complex of the Streptococcus thermophilus CRISPR3/Cas9 system introduces in vitro a double-strand break at a specific site in DNA containing a sequence complementary to crRNA. DNA cleavage is executed by Cas9, which uses two distinct active sites, RuvC and $\mathrm{HNH}$, to generate site-specific cuts on opposite DNA strands. Results demonstrate that the Cas9-crRNA complex functions as an RNA-guided endonuclease with RNA-directed target sequence recognition and protein-mediated DNA cleavage. These findings pave the way for engineering of universal programmable RNA-guided DNA endonucleases [16].

The revolution began when scientists figured out that the CRISPR system is programmable.

In 2012, Jennifer Dudna and Emanuela Sharpantier modified the Cas-9 enzyme to make it easier to work with. They create a variant of Cas-9, which uses single-stranded RNA in the CRISPR-Cas9 complex, which replaces crRNA and tracrRNA, which is called single guide RNA (sgRNA). sgRNA contains an RNA sequence complementary to a region of DNA that is desired to be excised. They were the first to propose that CRISPR/Cas9 could be used for programmable gene editing [17], which is considered as one of the most significant discoveries in the history of biology $[18,19]$. Their work has since been further developed by many research groups for applications ranging from fundamental 


\section{Phage infection}

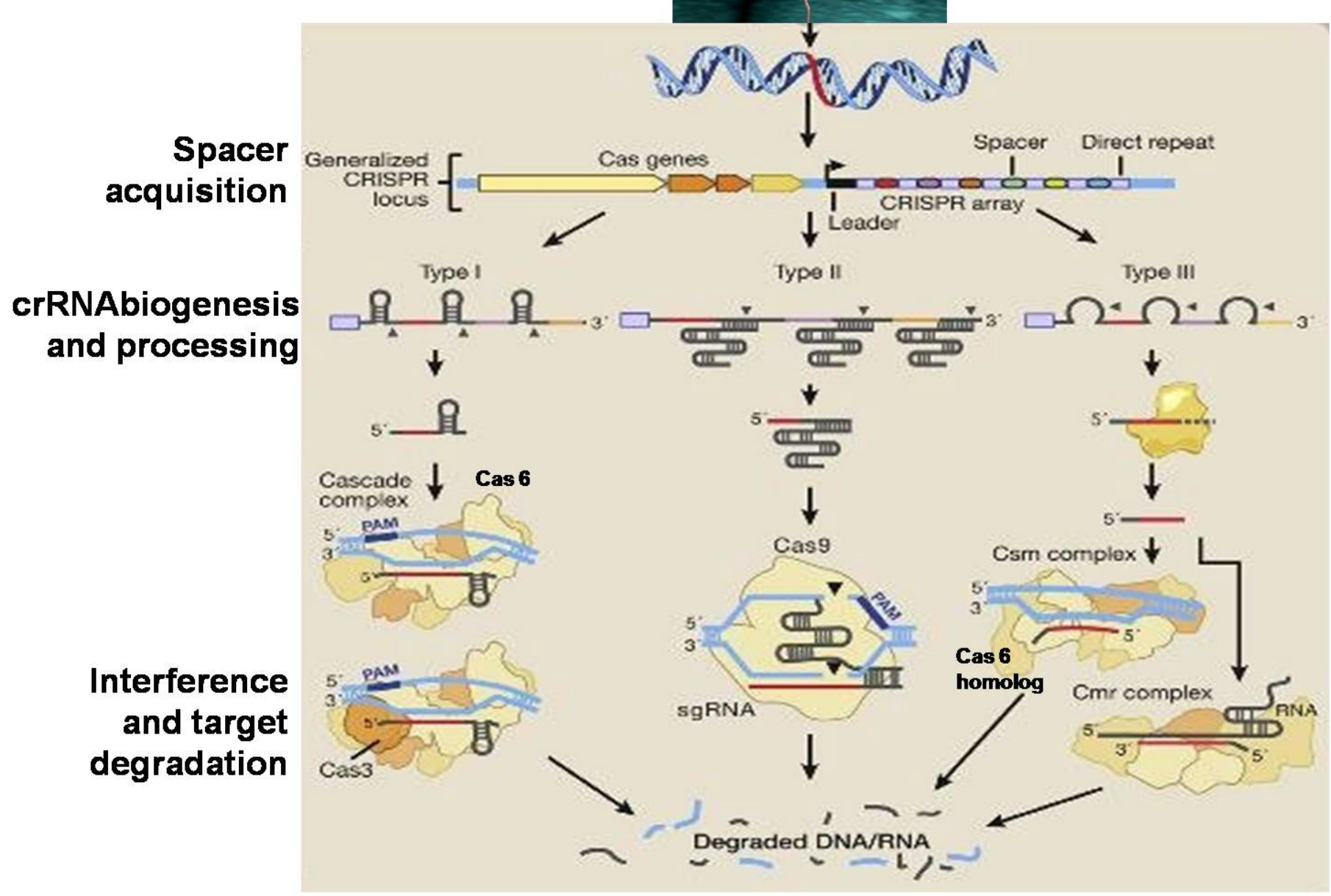

Figure 1. The CRISPR/Cas9 system. Cas9, one of the associated proteins, is an endonuclease that cuts both strands of DNA. Cas9 is directed to its target by a section of RNA. This can be synthesized as a single strand called a synthetic single guide RNA (sgRNA); the section of RNA which binds to the genomic DNA is 18-20 nucleotides. In order to cut, a specific sequence of DNA of between 2 and 5 nucleotides (the exact sequence depends upon the bacteria which produces the Cas9) must lie at the 3' end of the guide RNA: this is called the protospacer adjacent motif (PAM). Repair after the DNA cut may occur via two pathways: non-homologous end joining, typically leading to a random insertion/deletion of DNA, or homology directed repair where a homologous piece of DNA is used as a repair template. It is the latter which allows precise genome editing: the homologous section of DNA with the required sequence change may be delivered with the Cas9 nuclease and sgRNA, theoretically allowing changes as precise as a single base-pair

protein research to treatments for diseases including sickle cell anemia, cystic fibrosis, Huntington's disease, HIV, and others.

\section{Application}

Through Cas9 sgRNA, it is possible to make changes on a strictly specific gene. The primary role of this system is to deactivate genes by cutting the DNA molecule at a specific location. If Cas9 is modified it can acquire various functions. By inactivating nuclease activity and adding other proteins (deaminase), the Cas9 complex acquires the ability to replace only one nucleotide. This makes it possible to convert a mutated gene into its healthy form. Another possibility is complete inactivation of its nuclease activity and addition of transcriptional activators and thus enhance the transcription of a gene. The CRISPR/ Cas9 system can serve to inactivate/activate a gene, which may play a role in understanding its function. You can just give it a copy of DNA you want to modify and put the system into a living cell. When adding fluorescent proteins to Cas9, the spatial structure of the genome can be visualized as well as whole chromosome labeling. Another quality of the system is the ability to modify living cells such as somatic and germ line. Aside from being precise, cheap, and easy, CRISPR offers the ability to edit life cells, to switch genes on and off, and target and study particular DNA sequences. It also works for every type of cells: microorganisms, plants, animals, or humans.

In 2013 have been done heritable and precise editing of the zebrafish genome by CRISPR-Cas [20-23], on human cells [24-27].

The same year was efficiently targeted and modified by CRISPR/ Cas9 the genome of Drosophila [28,29], and plants [30,31].

In 2013 were published results that the CRISPR system can be used as a potential tool for the precise regulation of gene expression in eukaryotic cells [32-36].

Zebrafish has become a popular model organism to study human diseases as neurodegenerative diseases [37]. Zebrafish has become also 
a model for cardiac development and disease. The transparency of the embryo, its limited requirement for active oxygen delivery, and ease of use in genetic manipulations and chemical exposure have made it a powerful alternative to rodents. CRISPR-mediated genome engineering and advanced imaging methods will only accelerate its use [38].

CRISPR system has become a highly attractable approach for the study of genomic rearrangements as a powerful platform to manipulate the mammalian genome [39-41].

Recent advances in genome-editing techniques have made it possible to modify any desired DNA sequence by employing programmable nucleases. The $\beta$-haemoglobinopathies, such as sickle cell disease and $\beta$-thalassaemia, are caused by mutations in the $\beta$-globin gene and affect millions of people worldwide. Ex vivo gene correction in patient-derived haematopoietic stem cells followed by autologous transplantation could be used to cure $\beta$-haemoglobinopathies. These next-generation genome-modifying tools are the ideal candidates for therapeutic applications, especially for the treatment of genetic disorders like sickle cell anemy (SCA). SCA is an inheritable monogenic disorder which is caused by a point mutation in the $\beta$-globin gene. Substantial success has been achieved in the development of supportive therapeutic strategies for SCA, but unfortunately there is still a lack of long-term universal cure. The only existing curative treatment is based on allogeneic stem cell transplantation from healthy donors; however, this treatment is applicable to a limited number of patients only. Huang, et al. using CRISPR/Cas9 successfully corrected the endogenous Hbs point mutation in human induced pluripotent stem cells isolated from sickle cell disease patients and generate disease-free cells for treatment via transplantation back in the patients [42].

Hoban, et al. demonstrate correction of the sickle mutation in patient-derived CD34+ cells using CRISPR/Cas9 technology. Delivery of the CRISPR/Cas9 components to CD34+ cells led to over $18 \%$ gene modification in vitro and correction of the sickle cell disease mutation, leading to the production of wild-type hemoglobin [43].

Dever, et al. present a CRISPR/Cas9 gene-editing system that combines Cas9 ribonucleoproteins and adeno-associated viral vector delivery of a homologous donor to achieve homologous recombination at the $H b S$ gene in haematopoietic stem cells. They also propose a method to purify a population of haematopoietic stem and progenitor cells with more than $90 \%$ targeted integration and efficient correction of the Glu6Val mutation and express adult $\beta$-globin ( $\mathrm{HbA}$ ) messenger RNA [44]. In the study of Wen, et al. two separate point mutations 58T and 69A/70A were introduced as tracking markers in $H b S$ by CRISPR. Due to these genetic hallmarks, the resultant genome-engineered $H B B$ ( $\beta$-globin gene) was easily identified during sequencing. Importantly, the presence of two sequence hallmarks in $H B B$ ruled out the possibility of experimental contamination, unexpected genome variation, or artificial sequence mutations. Sequencing analysis demonstrated that these genetic hallmarks (58T and 70A) were always co-present and were detected in all genome-edited clones; although, the two mutation sites were more than 10 nucleotides apart. These findings indicate that it is possible to simultaneously edit two separate target sites in the same gene by the CRISPR/Cas9 approach, using a single pair of sgRNA and HDR template. Simultaneous genome editing of two target sites can be useful to treat diseases carrying two or more point mutation sites that are close to each other [45].

Using human induced pluripotent stem cells (iPSCs) from two $\beta$-thalassemia patients with different $H B B$ gene mutations, Cai, et al. 2018 devised and tested a universal strategy to achieve targeted insertion of the $H B B$ cDNA in exon 1 of $H B B$ gene using Cas9 and two validated guide RNAs. They observed that $H B B$ protein production was restored in erythrocytes derived from iPSCs of two patients. This strategy of restoring functional $H B B$ gene expression will be able to correct most types of $H B B$ gene mutations in $\beta$-thalassemia and SCA [46].

A CRYGC gene mutation is an autosomal dominant mutation that causes pulverulent cataract. After coinjection into mice zygotes of Cas 9 mRNA and a single-guide RNA (sgRNA) targeting the mutant allele correction occurred via homology-directed repair (HDR) based on an exogenously supplied oligonucleotide or the endogenous WT allele, with only rare evidence of off-target modifications. The resulting mice were fertile and able to transmit the corrected allele to their progeny. Thus, the study of $\mathrm{Wu}$, et al. provides proof of principle for use of the CRISPR-Cas9 system to correct genetic disease [47].

MYBPC3 is involved in hypertrophic cardiomyopathy a disease that makes the heart muscle thicker. It can lead to sudden heart failure and death in young, otherwise healthy athletes when they push their hearts too hard while exercising. The mutation is dominant, meaning that a child need inherit only one copy of the mutated gene to experience its effects. The disease can be managed with a variety of treatments, but there is no real cure. Recently reproductive biologist Shoukhrat Mitalipov and his team used genome editing to correct a gene that causes a potentially fatal heart condition in humans. An international team of researchers has used CRISPR-Cas9 gene editing to correct a disease-causing mutation in dozens of viable human embryos. If they fertilize an oocyte with spermatozoa carring mutant $M Y B P C 3$ together with Cas9 protein, sgRNA and DNA template, the fertilized eggs went to produce embryos with two healthy MYBPC3 genes in 42 out of 58 trials (over $70 \%$ of the cases) comparing the control experiment without any CRISPR were the result was around 50/50 [48].

Duchenne muscular dystrophy (DMD) is a severe muscledegenerative disease caused by a mutation in the dystrophin gene. Genetic correction of patient-derived induced pluripotent stem cells (iPSCs) by TALENs or CRISPR-Cas9 holds promise for DMD gene therapy; however, the safety of such nuclease treatment must be determined. To restore the dystrophin protein, Le, et al. performed three correction methods (exon skipping, frameshifting, and exon knockin) in DMD-patient-derived iPSCs, and found that exon knockin was the most effective approach. They differentiated the corrected iPSCs toward skeletal muscle cells and successfully detected the expression of full-length dystrophin protein [49].

Muscle stem cells (MuSCs) hold great therapeutic potential for muscle genetic disorders, such as Duchenne muscular dystrophy (DMD). Zhu, et al. used fibrin-gel culture system to selectively expand MuSCs from crude skeletal muscle cells of mdx mice, a mouse model of DMD. By CRISP/Cas9-based genome editing, they corrected the dystrophin mutation in expanded MuSCs and restored the skeletal muscle dystrophin expression upon transplantation in $\mathrm{mdx}$ mice [50].

These results provide an important framework for developing iPSC-based gene therapy for genetic disorders using programmable nucleases. Their studies established a reliable and feasible platform for gene correction in MuSCs by genome editing, thus greatly advancing tissue stem cell-based therapies for DMD and other muscle disorders.

Isolated human pancreatic islets are a rare and precious material for diabetes research. However, their availability is limited, and it is impossible to obtain them from patients with specific genotypes. Human pluripotent stem cells provide an alternative. Induced 
pluripotent stem cells can be generated from any individual's somatic cells and differentiated into pancreatic cells. Currently, this approach is limited by the immaturity of the islet-like cells derived from stem cells. However, this approach can already be used to model developmental defects, and the possibilities for studying insulin secretion are continuously improving. In addition, genome editing using the CRISPR/Cas9 technology provides powerful possibilities to study the impact of specific genotypes. The same technology can also be used for transcriptional regulation in order to improve the functional maturation of stem cell-derived islets. These tools are today becoming available for tomorrow's translational diabetes research. Using CRISPR Gimenez, et al. achieved activation of human insulin gene in fibroblasts isolated from patients with T1D [51]. The same year the first report demonstrating enhanced T1D in a mouse modeling human PTPN22 (R620W) and the utility of CRISPR-Cas9 for direct genetic alternation of NOD (non-obese diabetic) mice was published [52]. Using CRISPR/Cas9 in the LEW.1WR1 rat model of T1D the occurrence of Kilham rat virus-induced diabetes was diminished which support the use of strategies to limit or prevent the development of type 1 diabetes [53]. Ratiu, et al. found that CRISPR/Cas9-mediated ablation of the activation-induced cytidine deaminase gene required for class switch recombination/somatic hypermutation induction inhibits T1D development in the NOD mouse. The activation-induced cytidine deaminase protein induces genome-wide DNA breaks that, if not repaired through RAD51-mediated homologous recombination, result in B lymphocyte death. Treatment with the RAD51 inhibitor 4,4'-diisothiocyanatostilbene-2, 2'-disulfonic acid also strongly inhibited T1D development in NOD mice. The genetic and small molecule-targeting approaches expanded $\mathrm{CD}^{+} 3^{+} \mathrm{B}$ lymphocytes that exert regulatory activity suppressing diabetogenic $\mathrm{T}$ cell responses. Hence, an initial CRISPR/Cas9-mediated genetic modification approach has identified the $A I D / R A D 51$ axis as a target for a potentially clinically translatable pharmacological approach that can block T1D development by converting B lymphocytes to a disease-inhibitory $\mathrm{CD}^{+}{ }^{+}$regulatory state [54].

By CRISPR/Cas9 technology and pharmacological approaches were inactivated protein tyrosine phosphatases PTPN6 and PTPN1 in human islets and islets from NOD mice. The authors identify PTPN6 as a negative regulator of TNF- $\alpha$-induced $\beta$-cell death, through JNKdependent BCL-2 protein degradation. In contrast, PTPN1 acts as a positive regulator of IFN- $\gamma$-induced STAT1-dependent gene expression, which enhanced autoimmune destruction of $\beta$-cells. Importantly, PTPN1 inactivation by pharmacological modulation protects $\beta$-cells and primary mouse islets from cytokine-mediated cell death. These data point to a non-redundant effect of PTP regulation of cytokine signalling in $\beta$-cells in autoimmune diabetes [55].

By CRISPR/Cas9 mediated ZnT8 knock-out Merriman, et al. demonstrate that $Z n T 8$ is a cell surface self-antigen, raising the possibility of a direct involvement in antibody-mediated $\beta$-cell dysfunction and cytotoxicity [56]. By CRISPR-Cas9 technology have been investigated the function of GPRC5B receptor in $\beta$-Cells [57].

The CRISPR/Cas9 technology became an attractive for HIV tretment [58].

The HIV-1 Env glycoprotein is folded in the endoplasmic reticulum (ER), which is necessary for viral entry and replication. By CRISPR/ Cas9 was knocked out the TSPO gene in 293T cells and was found that TSPO could similarly inhibit Env expression in these cells [59].

Individuals homozygous for the $\mathrm{C}-\mathrm{C}$ chemokine receptor type 5 gene with 32-bp deletions (CCR5 $\Delta 32$ ) are resistant to HIV-1 infection.
Ye, at al. generated a pluripotent stem cells (iPSCs) homozygous for the naturally occurring CCR5 $\triangle 32$ mutation through genome editing of wild-type iPSCs using a combination of transcription activatorlike effector nucleases (TALENs) or RNA-guided clustered regularly interspaced short palindromic repeats (CRISPR)-Cas9 together with the piggyBac technology. These modified iPSCs into monocytes/ macrophages and demonstrated their resistance to HIV-1 challenge. Authors propose that this strategy may provide an approach toward a functional cure of HIV-1 infection [60].

CCR5 serves as an essential co-receptor for human immunodeficiency virus type 1 (HIV-1) entry, and individuals with a CCR5 $(\Delta 32)$ variant appear to be healthy, making CCR5 an attractive target for control of HIV-1 infection. CCR5, a co-receptor for HIV-1 entry, is a major target for drug and genetic intervention against HIV1. Wang, et al. silenced CCR5 via CRISPR/Cas9. They constructed lentiviral vectors expressing Cas9 and CCR5 sgRNAs. As a result CCR5 gene-disrupted cells are not only resistant to R5-tropic HIV-1, but also have selective advantage over CCR5 gene-undisrupted cells during R5-tropic HIV-1 infection. Silencing or disruption of CCR5 via Cas9 and CCR5-specific sgRNAs could be a viable alternative strategy for engineering resistance against HIV-1 [61,62]. Li, et al. constructing chimeric Ad5F35 adenoviruses carrying CRISPR/Cas9 components, efficiently transduced primary CD4(+) T-lymphocytes and disrupted CCR5 expression. The transduced primary CD4(+) T-cells were HIV-1 resistant [63].

Transplantation of hematopoietic stem cells (HSCs) with a naturally occurring CCR5 mutation confers a loss of detectable HIV-1 in the patient. Making ablation of the CCR5 gene in HSCs is an ideal therapy for an HIV-1 cure. Xu, et al. established a CRISPR/Cas9 gene editing system in human CD34+ HSPCs and achieved efficient CCR5 ablation which confers HIV-1 resistance in vivo. This strategy proposes a therapy by transplantation of CCR5 gene-edited HSC for an HIV cure to the clinic [64].

CXCR4 is a also co-receptor for the human immunodeficiency virus type 1 (HIV-1) infection and has been considered as an important therapeutic target for AIDS. CXCR4 mediates viral entry into human CD4(+) cells by binding to envelope protein, gp120. Human CXCR4 gene was efficiently disrupted by CRISPR/Cas9-mediated genome editing, leading to HIV-1 resistance of human primary CD4(+) T cells. The precise and efficient genome editing of CXCR4 will provide also a new strategy for therapeutic application against HIV-1 infection [65]. Recently, a small Staphylococcus aureus Cas9 (SaCas9) has been developed as a genome editing tool. It provides a promising strategy for HIV-1 gene therapy if it is used to target CXCR4. Wang, et al. demonstrated that transduction of lenti-virus expressing $\operatorname{SaCas} 9$ and selected single-guided RNAs of CXCR4 in human $\mathrm{CD}^{+} \mathrm{T}$ cell lines efficiently induced the editing of the CXCR4 gene and disrupted CXCR4 expression, making these cell lines resistant to X4-tropic HIV1 infection. CXCR4-edited primary $\mathrm{CD}^{+} \mathrm{T}$ cells proliferated normally and were resistant to HIV-1 infection [66].

Disruption of either the CCR5 or the CXCR4 gene confers resistance HIV-1 infection. However, the urgent need to ablate both of the co-receptors for dual protection remains. Yu, et al. ablated the CCR5 and CXCR4 genes in human CD4+ cell lines and primary CD4+ T cells simultaneously using CRISPR/Cas9. The results demonstrate the safety and efficacy of CRISPR/Cas9 in multiplex gene modification on peripherally circulating CD4+ T cells, which may promote a functional cure for HIV-1 infection [67]. Simultaneous genome editing of CXCR4 
and CCR5 by CRISPR-Cas9 can potentially provide an effective and safe strategy towards a functional cure for HIV-1 infection [68].

Gene editing using CRISPR/Cas9 may provide a new therapeutic path for eliminating HIV-1 DNA from CD4+ T-cells and potentially serve as a novel and effective platform toward curing AIDS [69].

CRISPR-Csy4 endoribonuclease is also a promising tool that could be tailored further to target HIV-1 [70].

A novel catalytically-deficient Cas9-synergistic activation mediator (dCas9-SAM) technology can serve as a novel HIV-latency-reversing therapeutic tool for the permanent elimination of HIV-1 latent reservoirs [71].

Gene-specific transcriptional activation can be achieved using the RNA-guided CRISPR-Cas9 system comprising single guide RNAs (sgRNAs) with a nuclease-deficient Cas9 mutant (dCas9) fused to the VP64 transactivation domain (dCas9-VP64). This system was engineered to target 23 sites within the long terminal repeat promoter of HIV-1 and identified a "hotspot" for activation within the viral enhancer sequence [72]. Also dCas9-SunTag-VP64 system can effectively and specifically reactivate latent HIV-1 transcription, suggesting that this strategy could offer a novel approach to anti-HIV-1 latency [73].

Complete eradication of HIV-1 infection is impeded by the existence of cells that harbor chromosomally integrated but transcriptionally inactive provirus. These cells can persist for years without producing viral progeny, rendering them refractory to immune surveillance and antiretroviral therapy and providing a permanent reservoir for the stochastic reactivation and reseeding of HIV-1. Strategies for purging this latent reservoir are thus needed to eradicate infection. CRISPR systems are potentially effective tools for inducing latent HIV-1 expression and in combination with antiretroviral therapy, could lead to improved therapies for HIV-1 infection [74].

Using HIV-1 infection as a model, Liao, et al. demonstrate that the CRISPR/Cas9 system disrupts latently integrated viral genome and provides long-term adaptive defense against new viral infection, expression and replication in human cells. These results unveil the potential of the CRISPR/Cas9 system as a new therapeutic strategy against viral infections [75].

Mutated by CRISPR/Cas9 target site in the second exon called T10 results in significantly diminished HIV-1 gene expression and virus production in latently infected Jurkat cells. That reveals a highly efficient Cas9 target site within the second exon of Rev that represents a promising target to be further explored in the CRISPR/Cas9-based cure strategy [76].

Kaminski, et al. demonstrate that functional activation of CRISPR/ Cas9 by Tat during the course of viral infection excises the designated segment of the integrated viral DNA and consequently suppresses viral expression [77].

A CRISPR/Cas9 gene editing strategy has been remarkable in infected human cell lines. Kaminski, et al. by tail-vein injection employed a short version of the Cas9 endonuclease, saCas 9 , together with a multiplex of guide RNAs (gRNAs) for targeting the HIV-1 DNA sequences within the 5'-LTR and the Gag gene for removing critically important segments in transgenic mice and rats encompassing the HIV-1 genome. These results for the first time, demonstrate the in vivo eradication of HIV-1 DNA by CRISPR/Cas9 [78]. In vivo excision of HIV-1 proviral DNA by $s g R N A s / s a C a s 9$ in solid tissues/organs in mice can be achieved via AAV delivery, a significant step toward human clinical trials [79].

Astrocytes, the most abundant cells in the mammalian brain, perform key functions and are involved in several neurodegenerative diseases. The human immunodeficiency virus (HIV) can persist in astrocytes, contributing to the HIV burden and neurological dysfunctions in infected individuals. Huang and Nair established a novel, simple and quick screening method to identify gRNA candidates for targeting HIV provirus in astrocytes [80]. A novel adeno-associated virus-based vector ( $A A V 9 P 1)$ with a synthetic surface peptide for transduction of astrocytes was reported as an instrument to deliver $\mathrm{HIV}$-inhibitory genes to astrocytes. Kunze, et al. generated $A A V 9 P 1$ vectors containing genes for HIV-1 proviral editing by CRISPR/Cas9. Latently HIV-1 infected astrocytes transduced with these vectors showed significantly diminished reactivation of proviruses, compared with untransduced cultures. AAV9P1 is a promising tool for gene delivery to astrocytes and may facilitate inactivation/destruction of persisting HIV-1 proviruses in astrocyte reservoirs [81].

To date, anti-HIV-1 gRNAs have been designed target certain sequence, however, they seldom account for genetic variation in the HIV-1 genome within and between patients, which will be crucial for therapeutic application of this technology. Dampier, et al. created a basic computational tools for analyzing the patient-derived HIV-1 sequences to give information for gRNA design [82].

The type I interferon (IFN-I)-inducible human restriction factor TRIM $5 \alpha$ inhibits the infection of human cells by specific nonhuman retroviruses, such as N-MLV and EIAV, but does not generally target HIV-1. However, the introduction of two aminoacid substitutions, $R 332 G$ and $R 355 G$, in the human TRIM5a (huTRIM5 $\alpha$ ) domain responsible for retroviral capsid recognition leads to efficient HIV1 restriction upon stable over-expression. By CRISPR-Cas9 was successfully mutated TRIM5 to its potentially HIV-1-restrictive version by homology-directed repair (HDR) in HEK293T cells. The TRIM5 gene editing identifies a new challenge in order to use this approach to confer protection from HIV-1 [83].

Experimental depletion of USP18 by CRISPR-Cas9 results in a significant restriction of HIV-1 replication in an induced pluripotent stem cell (iPSC)-derived macrophage model [84].

The HIV-1 suppression by CRISPR/Cas9 has advantages and disadvantages. Early RNAi experiments demonstrated profound virus inhibition, but also indicated that viral escape is possible. There are observations that illustrate opposite results of the effect of CRISPR/ Cas9 in inactivating HIV-1 which potentially restricting the use of CRISPR/Cas9 in HIV-1 therapy [85-88]. HIV virus may produce CRISPR/Cas9 resistant strains [89].

There are potential difficulties that must be addressed when considering anti-HIV-1 treatment with the CRISPR/Cas9 system alone. HIV-1 can escape from a single antiviral gRNA by mutation of the target sequence. Combinations of two antiviral gRNAs delay viral escape [90,91].

But sometime must be very carefully with combinatorial approaches also [92].

In a few decades, a CRISPR therapy might cure HIV and retroviruses, viruses that hide inside human DNA like could be eradicated [93-100] and for a cancer therapy [101-105].

RNA-programmed Cas 9 could offer considerable potential for genome editing in cells of the three kingdoms of life for 
biotechnological, biomedical and gene-therapeutic purposes. CRISPR/ Cas9 might help in understanding of functions of genes and unravel new pathways, engineering of disease models, screening for new targets for therapeutics, genetic predisposition for diseases, gene therapy. Attention is needed to dissolve some risks of CRISPR/Cas9 induced indel mutations [106,107].

Ding, et al. report that the repair of the on- and off-target cleavage resulted in a wide variety of insertions, deletions and point mutations. Therefore, CRISPR/Cas9 systems need to be carefully designed to avoid unexpected cleavage sites. Being effective in repairing variety of point mutations and insertions, genome editing with the CRISPR/Cas9 system disrupts the Pcsk9 gene in vivo with high efficiency and reduces blood cholesterol levels in mice. This approach may have therapeutic potential for the prevention of cardiovascular disease in humans [108].

The CRISPR/Cas RNA-guided cleavage of the target DNA, is a powerful approach for targeted gene disruption in various animal models [109].

However, there is little verification of microinjection methods for generating knockout mice using this approach. Horii, et al. compared three methods for injection: (1) injection of DNA into the pronucleus, (2) injection of RNA into the pronucleus, and (3) injection of RNA into the cytoplasm. They found that injection of RNA into the cytoplasm was the most efficient method in terms of the numbers of viable blastocyst stage embryos and full-term pups generated. This method also showed the best overall knockout efficiency [110].

\section{Ethical issues}

This technology is an extremely good weapon in the treatment of genetic diseases and viruses in the human genome. In order for these changes to remain lasting and to pass on to the next generation, it is necessary to modify genes or embryos. But the case arises: if it is possible to change the human genome to treat illnesses, why it is not possible to change the features of the fetus at the wish of the parents. If this is allowed, it is possible that the variety of genes in the genomic pool will decrease and the variety of the human species will disappear. Another option is to reverse the aging process and eliminate elderly diseases.

World without diseases? A potentially powerful application of gene drives: the development of disease-resistant mosquitoes that could knock out Malaria and Zika.

Gene editing can now change an entire species forever. CRISPR gene drives allow scientists to change sequences of DNA and guarantee that the resulting edited genetic trait is inherited by future generations, opening up the possibility of altering entire species forever. More than anything, this technology has led to questions: How will this new power affect humanity? What are we going to use it to change? Are we gods now? The future for CRISPR will be the new age for mankind. Genetic engineering will change everything forever.

Because this technique can do a lot of genetic engineering stuff, and it is acceptable to a lot of people. And mostly that is just really exciting.

CRISPR is a very powerful tool that needs a lot of contemplation about its consequences one tiny mistake and this could lead to a catastrophe.

Except to treatment of diseases CRISPR can and probably will be used for much more: the creation of modified humans - designer babies - and will mean gradual, but irreversible changes to the human gene pool. The first designed babies will be created to eliminate a deadly genetic disease running in a family. As the technology progresses and gets more refined, more and more people will use it. As soon as the first engineered kid is born, a door is opened and can't be closed any more. We know from nature that there are animals immune to aging. May be we could even borrow a few genes from them. The challenges are enormous. Designer babies, the end of diseases, genetically modified humans that never age. Outrageous things that used to be science fiction are suddenly becoming reality. The only thing we know for sure is that things will change irreversibly. But despite of revolution CRISPR/Cas9 now is for science, it is still just a first generation tool.

\section{Conclusions}

CRISPR is changing the entire field of biology. Using this technique, scientists can edit genes with a precision, accuracy and speed they never had before. It is possible to change the number of some specific genes in a cell, or delete it completely. It is possible also make a mutation in a gene, by altering, adding, or taking out pieces of DNA or single base pairs. Or you can introduce a gene from totally different organism. Like how we make bacteria that synthesize insulin, and we use the gene for GFP, the glowing green protein from jellyfish, as a biomarker in lots of experiments. There are a handful of techniques, but none of them is such convenient. For example by transfection the cells will express the gene for a few days, but you get too much of the gene floating around. Plus it is not part of a chromosome of other genes and regulatory bits, so you cannot be sure it is behaving normally. You can also use a modified virus to deliver a gene, because they normally integrate their genetic material in the hosts DNA. However, this method has problems too: the virus does not care where it sticks the new gene, so it could be smack in the middle of another gene and cause a bunch of problems. Changing a whole organism, like knocking out a gene in a mice, can take a years, thousands of dollars, generations of breeding and bit of luck. CRISPR/Cas9 is a system where you can send one protein to make a precise cut anywhere in the genome, just by giving it a piece of RNA. The cell uses a template to repair DNA. It is possible to send in a template along with the RNA and Cas9, so it adds whatever you want, like a whole new gene. Since the templates guided to a specific part of the genome, you are going to have just the right amount of your gene, integrated into a chromosome - unlike older techniques, where you get way too many copies, or even too few. So CRISPR/Cas9 makes gene editing faster, cheaper, and more accurate than even before.

But like any technique, the CRISPR/Cas9 system is not perfect. Sometimes, it cuts in the wrong place. It might stick at a sequence that almost matches to the guide RNA. In lab-grown cells this is not a huge problem. It is possible to use the technique a couple of times and check for cell that do it right, and it is still way better than inaccuracies from other methods. But doctors are also beginning to develop CRISPR/ Cas9 as a gene therapy for patients. Making a wrong cut in the genome of an already sick human could be disastrous. If they have cancer, for instance, an extra damaged gene in the already mutated cells might make the disease worse. That is why scientists have been carefully engineering the guide RNA and the amino acid sequence of Cas9 to try and make the technique more accurate, and cut the right sequence every time. They have made enough progress that human clinical trials involving CRISPR/Cas9 are making their way through the approval process: one at the University of Pennsylvania in the US and one at Sichuan University in China. Both are combining CRISPR/Cas9 with another hot cancer treatment - immunotherapy. Both trials will take some of the patient's immune cells, and use CRISPR/Cas9 to give them a genetic cancer-spotting boost. Than the immune cells will be turning 
back in the patient so they can get to work. The researchers expect CRISPR/Cas9 method to work even better. Except editing an adult's genes in their one cells CRISPR/Cas9 gives scientists the power to edit human embryos.

They are also trials using CRISPR/Cas9 to kill or to alter the genome of malaria transmitting mosquitoes not to be able to spread the malaria plasmid.

But if humans can now manipulate the genome of any organism, there are ethical questions that we need to consider. It is expected that the rapid advances in CRISPR/Cas9 technology will reduce and ultimately eliminate the risk of off-target gene effects, and CRISPR/ Cas9 will become the standard method to cure genetic disorders in routine clinical practice.

For now CRISPR/Cas9 is mostly being used for editing genomes in cells at cell cultures to learn how they function without a gene, or with a new one.

\section{References}

1. Ishino Y, Shinagawa H, Makino K, Amemura M, Nakata A (1987) "Nucleotide sequence of the iap gene, responsible for alkaline phosphatase isozyme conversion in Escherichia coli, and identification of the gene product”. J Bacteriol 169: 5429-5433.

2. Mojica FJ, Juez G, Rodriguez-Valera F (1993) Transcription at different salinities of Haloferax mediterranei sequences adjacent to partially modified PstI sites. Mol Microbiol 9: 613-621.

3. Jansen R, Embden JD, Gaastra W, Schouls LM (2002) Identification of genes that are associated with DNA repeats in prokaryotes. Mol Microbiol 43: 1565-1575. [Crossref]

4. Bolotin A, Quinquis B, Sorokin A, Ehrlich SD (2005) Clustered regularly interspaced short palindrome repeats (CRISPRs) have spacers of extrachromosomal origin. Microbiology 151: 2551-2561.

5. Barrangou R, Fremaux C, Deveau H, Richards M, Boyaval P, et al. (2007) CRISPR provides acquired resistance against viruses in prokaryotes. Science 315: 1709-1712.

6. Marraffini LA, Sontheimer EJ (2009) Invasive DNA, chopped and in the CRISPR. Structure 17: 786-788. [Crossref]

7. van der Oost J, Jore MM, Westra ER, Lundgren M, Brouns SJ (2009) CRISPR-based adaptive and heritable immunity in prokaryotes. Trends Biochem Sci 34: 401-407. [Crossref]

8. Horvath P, Barrangou R (2010) CRISPR/Cas, the immune system of bacteria and archaea. Science 327: 167-170. [Crossref]

9. Hale CR, Zhao P, Olson S, Duff MO, Graveley BR, et al. (2009) RNA-guided RNA cleavage by a CRISPR RNA-Cas protein complex. Cell 139: 945-956. [Crossref]

10. Marraffini LA, Sontheimer EJ (2010) Self versus non-self discrimination during CRISPR RNA-directed immunity. Nature 463: 568-571. [Crossref]

11. Marraffini LA, Sontheimer EJ (2010) CRISPR interference: RNA-directed adaptive immunity in bacteria and archaea. Nat Rev Genet 11: 181-190. [Crossref]

12. Karginov FV, Hannon GJ (2010) The CRISPR system: small RNA-guided defense in bacteria and archaea. Mol Cell 37: 7-19. [Crossref]

13. Garneau JE, Dupuis MĖ, Villion M, Romero DA, Barrangou R, et al. (2010) The CRISPR/Cas bacterial immune system cleaves bacteriophage and plasmid DNA. Nature 468: 67-71. [Crossref]

14. Babu M, Beloglazova N, Flick R, Graham C, Skarina T, et al. (2011) A dual function of the CRISPR-Cas system in bacterial antivirus immunity and DNA repair. Mol Microbiol 79: 484-502. [Crossref]

15. Sapranauskas R, Gasiunas G, Fremaux C, Barrangou R, Horvath P, et al. (2011) The Streptococcus thermophilus CRISPR/Cas system provides immunity in Escherichia coli. Nucleic Acids Res 39: 9275-9282.

16. Gasiunas G, Barrangou R, Horvath P, Siksnys V (2012) Cas9-crRNA ribonucleoprotein complex mediates specific DNA cleavage for adaptive immunity in bacteria. Proc Natl Acad Sci USA 109: E2579-E2586.

17. Jinek M, Chylinski K, Fonfara I, Hauer M, Doudna JA, et al. (2012) A programmable dual-RNA-guided DNA endonuclease in adaptive bacterial immunity. Science 337: 816-821. [Crossref]

18. Pollack, Andrew (2015) Jennifer Doudna, a Pioneer Who Helped Simplify Genome
Editing". New York Times.

19. Barrangou R, Doudna JA (2016) Applications of CRISPR technologies in research and beyond. Nat Biotechnol 34: 933-941. [Crossref]

20. Hwang WY, Fu Y, Reyon D, Maeder ML, Tsai SQ, et al. (2013) Efficient genome editing in zebrafish using a CRISPR-Cas system. Nat Biotechnol 31: 227-229. [Crossref]

21. Hwang WY, Fu Y, Reyon D, Maeder ML, Kaini P, et al. (2013) Heritable and precise zebrafish genome editing using a CRISPR-Cas system. PLoS One 8: e68708. [Crossref]

22. Ota S, Hisano Y, Ikawa Y, Kawahara A (2014) Multiple genome modifications by the CRISPR/Cas9 system in zebrafish. Genes Cells 19: 555-564. [Crossref]

23. Kimura Y, Hisano Y, Kawahara A, Higashijima S (2014) Efficient generation of knockin transgenic zebrafish carrying reporter/driver genes by CRISPR/Cas9-mediated genome engineering. Sci Rep 4: 6545.

24. Cho SW, Kim S, Kim JM, Kim JS (2013) Targeted genome engineering in human cells with the Cas9 RNA-guided endonuclease. Nat Biotechnol 31: 230-232. [Crossref]

25. Jinek M, East A, Cheng A, Lin S, Ma E, et al. (2013) RNA-programmed genome editing in human cells. Elife 2: e00471. [Crossref]

26. Fu Y, Foden JA, Khayter C, Maeder ML, Reyon D, et al. (2013) High-frequency off-target mutagenesis induced by CRISPR-Cas nucleases in human cells. Nat Biotechnol 31: 822-826. [Crossref]

27. Maeder ML, Linder SJ, Cascio VM, Fu Y, Ho QH, et al. (2013) CRISPR RNA-guided activation of endogenous human genes. Nat Methods 10: 977-979. [Crossref]

28. Bassett AR, Tibbit C, Ponting CP, Liu JL (2013) Highly efficient targeted mutagenesis of Drosophila with the CRISPR/Cas9 system. Cell Rep 4: 220-228.

29. Yu Z, Ren M, Wang Z, Zhang B, Rong YS, et al. (2013) Highly efficient genome modifications mediated by CRISPR/Cas9 in Drosophila. Genetics 195: 289-291.

30. Xie K, Yang Y (2013) RNA-guided genome editing in plants using a CRISPR-Cas system. Mol Plant 6: 1975-1983. [Crossref]

31. Feng Z, Zhang B, Ding W, Liu X, Yang DL, et al. (2013) Efficient genome editing in plants using a CRISPR/Cas system. Cell Res 23: 1229-1232. [Crossref]

32. Gilbert LA, Larson MH, Morsut L, Liu Z, Brar GA, et al. (2013) CRISPR-mediated modular RNA-guided regulation of transcription in eukaryotes. Cell 154: 442-451. [Crossref]

33. Mao Y, Zhang H, Xu N, Zhang B, Gou F, et al. (2013) Application of the CRISPR-Cas system for efficient genome engineering in plants. Mol Plant 6: 2008-2011. [Crossref]

34. Walsh RM, Hochedlinger K (2013) A variant CRISPR-Cas9 system adds versatility to genome engineering. Proc Natl Acad Sci USA 110: 15514-15515. [Crossref]

35. Zhonggang Hou, Yan Zhang, Nicholas EP, Sara EH, Li-Fang Chu, et al. (2013) Efficient genome engineering in human pluripotent stem cells using Cas9 from Neisseria meningitides. Proc Natl Acad Sci USA 110: 15644-15649.

36. Wu X, Scott DA, Kriz AJ, Chiu AC, Hsu PD, et al. (2014) Genome-wide binding of the CRISPR endonuclease Cas9 in mammalian cells. Nat Biotechnol 32: 670-676. [Crossref]

37. Schmid B, Haass C (2013) Genomic editing opens new avenues for zebrafish as a model for neurodegeneration. $J$ Neurochem 127: 461-470. [Crossref]

38. Wilkinson RN, Jopling C, van Eeden FJ (2014) Zebrafish as a model of cardiac disease. Prog Mol Biol Transl Sci 124: 65-91. [Crossref]

39. Choi PS, Meyerson M (2014) Targeted genomic rearrangements using CRISPR/Cas technology. Nat Commun 5: 3728. [Crossref]

40. Horii T, Morita S, Kimura M, Kobayashi R, Tamura D, et al. (2013) Genome engineering of mammalian haploid embryonic stem cells using the Cas9/RNA system. PeerJ 1: e230.

41. Hsu PD, Lander ES, Zhang F (2014) Development and applications of CRISPR-Cas9 for genome engineering. Cell 157: 1262-1278. [Crossref]

42. Huang X, Wang Y, Yan W, Smith C, Ye Z, et al. (2015) Production of Gene-Corrected Adult Beta Globin Protein in Human Erythrocytes Differentiated from Patient iPSCs After Genome Editing of the Sickle Point Mutation. Stem Cells 33: 1470-1479.

43. Hoban MD, Lumaquin D, Kuo CY, Romero Z, Long J, et al. (2016) CRISPR/Cas9Mediated Correction of the Sickle Mutation in Human CD34+ cells. Mol Ther 24: 1561-1569. [Crossref] 
44. Dever DP, Bak RO, Reinisch A, Camarena J, Washington G, et al. (2016) CRISPR/ Cas9 $\hat{\mathrm{I}}^{2}$-globin gene targeting in human haematopoietic stem cells. Nature 539: 384389. [Crossref]

45. Wen J, Tao W, Hao S, Zu Y (2017) Cellular function reinstitution of offspring red blood cells cloned from the sickle cell disease patient blood post CRISPR genome editing. J Hematol Oncol 10: 119.

46. Cai L, Bai H (2018) A Universal Approach to Correct Various HBB Gene Mutations in Human Stem Cells for Gene Therapy of Beta-Thalassemia and Sickle Cell Disease. Stem Cells Transl Med 7: 87-97. [Crossref]

47. Wu Y, Liang D, Wang Y, Bai M, Tang W, et al. (2013) Correction of a genetic disease in mouse via use of CRISPR-Cas9. Cell Stem Cell 13: 659-662. [Crossref]

48. Ma H, Marti-Gutierrez N, Park SW, Wu J, Lee Y, et al. (2017) Correction of a pathogenic gene mutation in human embryos. Nature 548: 413-419. [Crossref]

49. Li HL, Fujimoto N, Sasakawa N, Shirai S, Ohkame T, et al. (2015) Precise correction of the dystrophin gene in duchenne muscular dystrophy patient induced pluripotent stem cells by TALEN and CRISPR-Cas9. Stem Cell Reports 4: 143-154.

50. Zhu P, Wu F, Mosenson J, Zhang H, He TC, et al. (2017) CRISPR/Cas9-Mediated Genome Editing Corrects Dystrophin Mutation in Skeletal Muscle Stem Cells in a Mouse Model of Muscle Dystrophy. Mol Ther Nucleic Acids 7: 31-41.

51. Giménez CA, Ielpi M, Mutto A, Grosembacher L, Argibay P, et al. (2016) CRISPR-on system for the activation of the endogenous human INS gene. Gene Ther 23: 543-547. [Crossref]

52. Lin X, Pelletier S, Gingras S, Rigaud S, Maine CJ, et al. (2016) CRISPR-Cas9Mediated Modification of the NOD Mouse Genome With Ptpn22R619W Mutation Increases Autoimmune Diabetes. Diabetes 65: 2134-2138.

53. Qaisar N, Lin S, Ryan G, Yang C, Oikemus SR, et al. (2017) A Critical Role for the Type I Interferon Receptor in Virus-Induced Autoimmune Diabetes in Rats. Diabetes 66: 145-157. [Crossref]

54. Ratiu JJ, Racine JJ, Hasham MG, Wang Q, Branca JA, et al. (2017) Genetic and Smal Molecule Disruption of the AID/RAD51 Axis Similarly Protects Nonobese Diabetic Mice from Type 1 Diabetes through Expansion of Regulatory B Lymphocytes. $J$ Immunol 198: 4255-4267.

55. Stanley WJ, Trivedi PM, Sutherland AP, Thomas HE, Gurzov EN (2017) Differentia regulation of pro-inflammatory cytokine signalling by protein tyrosine phosphatases in pancreatic ß-cells. J Mol Endocrinol 59: 325-337.

56. Merriman C, Huang Q, Gu W, Yu L, Fu D (2018) A subclass of serum anti-ZnT8 antibodies directed to the surface of live pancreatic $\hat{I}^{2}$-cells. J Biol Chem 293: 579-587. [Crossref]

57. Atanes P, Ruz-Maldonado I, Hawkes R, Liu B, Persaud SJ, et al. (2018) Identifying Signalling Pathways Regulated by GPRC5B in B-Cells by CRISPR-Cas9-Mediated Genome Editing. Cell Physiol Biochem 45: 656-666.

58. Hu W, Kaminski R, Yang F, Zhang Y, Cosentino L, et al. (2014) RNA-directed gene editing specifically eradicates latent and prevents new HIV-1 infection. Proc Natl Acad Sci USA 111: 11461-11466.

59. Zhou T, Dang Y, Zheng YH (2014) The mitochondrial translocator protein, TSPO, inhibits HIV-1 envelope glycoprotein biosynthesis via the endoplasmic reticulumassociated protein degradation pathway. $J$ Virol 88: 3474-3484.

60. Ye L, Wang J, Beyer AI, Teque F, Cradick TJ, et al. (2014) Seamless modification of wild-type induced pluripotent stem cells to the natural CCR5?32 mutation confers resistance to HIV infection. Proc Natl Acad Sci USA 111: 9591-9596.

61. Wang W, Ye C, Liu J, Zhang D, Kimata JT, et al. (2014) CCR5 gene disruption via lentiviral vectors expressing Cas9 and single guided RNA renders cells resistant to HIV-1 infection. PLoS One 9: e115987.

62. Kang H, Minder P, Park MA, Mesquitta WT, Torbett BE, et al. (2015) CCR5 Disruption in Induced Pluripotent Stem Cells Using CRISPR/Cas9 Provides Selective Resistance of Immune Cells to CCR5-tropic HIV-1 Virus. Mol Ther Nucleic Acids 4: e268.

63. Li C, Guan X, Du T, Jin W, Wu B, et al. (2015) Inhibition of HIV-1 infection of primary CD4+ T-cells by gene editing of CCR5 using adenovirus-delivered CRISPR/ Cas9. J Gen Virol 96: 2381-2393. [Crossref]

64. Xu L, Yang H, Gao Y, Chen Z, Xie L, et al. (2017) CRISPR/Cas9-Mediated CCR5 Ablation in Human Hematopoietic Stem/Progenitor Cells Confers HIV-1 Resistance In Vivo. Mol Ther 25: 1782-1789. [Crossref]

65. Hou P, Chen S, Wang S, Yu X, Chen Y, Jiang M, Zhuang K, Ho W, Hou W, Huang J, Guo D. (2015) Genome editing of CXCR4 by CRISPR/cas9 confers cells resistant to HIV-1 infection. Sci Rep 5: 15577.
66. Wang Q, Chen S, Xiao Q, Liu Z, Liu S, et al. (2017) Genome modification of CXCR4 by Staphylococcus aureus Cas9 renders cells resistance to HIV-1 infection. Retrovirology 14: 51.

67. Yu S, Yao Y, Xiao H, Li J, Liu Q, et al. (2018) Simultaneous Knockout of CXCR4 and CCR5 Genes in CD4+ T Cells via CRISPR/Cas9 Confers Resistance to Both X4and R5-Tropic Human Immunodeficiency Virus Type 1 Infection. Hum Gene Ther 29: 51-67.

68. Liu Z, Chen S, Jin X, Wang Q (2017) Genome editing of the HIV co-receptors CCR5 and CXCR4 by CRISPR-Cas9 protects CD4+ T cells from HIV-1 infection. Cell Biosci 7: 47. [Crossref]

69. Kaminski R, Chen Y, Fischer T, Tedaldi E, Napoli A, et al. (2016) Elimination of HIV-1 Genomes from Human T-lymphoid Cells by CRISPR/Cas9 Gene Editing. Sci Rep 6: 22555.

70. Guo R, Wang H, Cui J, Wang G, Li W, et al. (2015) Inhibition of HIV-1 Viral Infection by an Engineered CRISPR Csy4 RNA Endoribonuclease. PLoS One 10: e0141335. [Crossref]

71. Zhang Y, Yin C, Zhang T, Li F, Yang W, et al. (2015) CRISPR/gRNA-directed synergistic activation mediator (SAM) induces specific, persistent and robust reactivation of the HIV-1 latent reservoirs. Sci Rep 5: 16277.

72. Saayman SM, Lazar DC, Scott TA, Hart JR (2016) Potent and Targeted Activation of Latent HIV-1 Using the CRISPR/dCas9 Activator Complex. Mol Ther 24: 488-498. [Crossref]

73. Ji H, Jiang Z, Lu P, Ma L, Li C (2016) Specific Reactivation of Latent HIV-1 by dCas9SunTag-VP64-mediated Guide RNA Targeting the HIV-1 Promoter. Mol Ther 24: 508521. [Crossref]

74. Limsirichai P, Gaj T, Schaffer DV (2016) CRISPR-mediated Activation of Latent HIV1 Expression. Mol Ther 24: 499-507. [Crossref]

75. Liao HK, Gu Y, Diaz A, Marlett J, Takahashi Y, et al. (2015) Use of the CRISPR Cas9 system as an intracellular defense against HIV-1 infection in human cells. Nat Commun 6: 6413. [Crossref]

76. Zhu W, Lei R, Le Duff Y, Li J, Guo F, et al. (2015) The CRISPR/Cas9 system inactivates latent HIV-1 proviral DNA. Retrovirology 12: 22. [Crossref]

77. Kaminski R, Chen Y, Salkind J, Bella R, Young WB, et al. (2016) Negative Feedback Regulation of HIV-1 by Gene Editing Strategy. Sci Rep 6: 31527. [Crossref]

78. Kaminski R, Bella R, Yin C, Otte J, Ferrante P, et al. (2016) Excision of HIV-1 DNA by gene editing: a proof-of-concept in vivo study. Gene Ther 23: 690-695.

79. Yin C, Zhang T, Qu X, Zhang Y, Putatunda R, et al. (2017) In Vivo Excision of HIV1 Provirus by saCas9 and Multiplex Single-Guide RNAs in Animal Models. Mol Ther 25: 1168-1186. [Crossref]

80. Huang Z, Nair M (2017) A CRISPR/Cas9 guidance RNA screen platform for HIV provirus disruption and HIV/AIDS gene therapy in astrocytes. Sci Rep 7: 5955. [Crossref]

81. Kunze C, Börner K, Kienle E (2018) Synthetic AAV/CRISPR vectors for blocking HIV-1 expression in persistently infected astrocytes. Glia 66: 413-427. [Crossref]

82. Dampier W, Sullivan NT (2017) Designing broad-spectrum anti-HIV-1 gRNAs to target patient-derived variants. Sci Rep 7: 14413. [Crossref]

83. Dufour C, Claudel A, Joubarne N, Merindol N, Maisonnet T, et al. (2018) Editing of the human TRIM5 gene to introduce mutations with the potential to inhibit HIV-1. PLoS One 13: e0191709. [Crossref]

84. Taylor JP, Cash MN, Santostefano KE, Nakanishi M (2018) CRISPR/Cas9 knockout of USP18 enhances type I IFN responsiveness and restricts HIV-1 infection in macrophages. J Leukoc Biol. [Crossref]

85. Wang Z, Pan Q, Gendron P, Zhu W, Guo F, et al. (2016) CRISPR/Cas9-Derived Mutations Both Inhibit HIV-1 Replication and Accelerate Viral Escape. Cell Rep 15 481-489. [Crossref]

86. Wang G, Zhao N, Berkhout B, Das AT (2016) CRISPR-Cas9 Can Inhibit HIV-1 Replication but NHEJ Repair Facilitates Virus Escape. Mol Ther 24: 522-526. [Crossref]

87. Ueda S, Ebina H, Kanemura Y, Misawa N, Koyanagi Y (2016) Anti-HIV-1 potency of the CRISPR/Cas9 system insufficient to fully inhibit viral replication. Microbiol Immunol 60: 483-496.

88. Herrera-Carrillo E, Berkhout B (2016) Attacking HIV-1 RNA versus DNA by sequence-specific approaches: RNAi versus CRISPR-Cas. Biochem Soc Trans 44 1355-1365. [Crossref] 
89. Yoder KE, Bundschuh R (2016) Host Double Strand Break Repair Generates HIV-1 Strains Resistant to CRISPR/Cas9. Sci Rep 6: 29530. [Crossref]

90. Wang G, Zhao N, Berkhout B, Das AT (2016) A Combinatorial CRISPR-Cas9 Attack on HIV-1 DNA Extinguishes All Infectious Provirus in Infected T Cell Cultures. Cell Rep 17: 2819-2826. [Crossref]

91. Lebbink RJ, de Jong DC, Wolters F, Kruse EM, van Ham PM, et al. (2017) A combinational CRISPR/Cas9 gene-editing approach can halt HIV replication and prevent viral escape. Sci Rep 7: 41968. [Crossref]

92. Zhao N, Wang G, Das AT, Berkhout B (2017) Combinatorial CRISPR-Cas9 and RNA Interference Attack on HIV-1 DNA and RNA Can Lead to Cross-Resistance. Antimicrob Agents Chemother 61: e01486- e015117.

93. Schiffer JT, Swan DA, Stone D, Jerome KR (2013) Predictors of hepatitis B cure using gene therapy to deliver DNA cleavage enzymes: a mathematical modeling approach. PLoS Comput Biol 9: e1003131. [Crossref]

94. Zhen S, Hua L, Takahashi Y, Narita S, Liu YH, et al. (2014) In vitro and in vivo growth suppression of human papillomavirus 16-positive cervical cancer cells by CRISPR Cas9. Biochem Biophys Res Commun 450: 1422-1426.

95. White MK, Hu W, Khalili K (2015) The CRISPR/Cas9 genome editing methodology as a weapon against human viruses. Discov Med 19: 255-262.

96. Wollebo HS, Bellizzi A, Kaminski R, Hu W, White MK, et al. (2015) CRISPR Cas9 System as an Agent for Eliminating Polyomavirus JC Infection. PLoS One 10: e0136046. [Crossref]

97. Bogerd HP, Kornepati AV, Marshall JB, Kennedy EM, Cullen BR (2015) Specific induction of endogenous viral restriction factors using CRISPR/Cas-derived transcriptional activators. Proc Natl Acad Sci USA 112: E7249-E7256.

98. Choi JG, Dang Y, Abraham S, Ma H, Zhang J, et al. (2016) Lentivirus pre-packed with Cas9 protein for safer gene editing. Gene Ther 23: 627-633. [Crossref]

99. Wahid B, Usman S, Ali A, Saleem K, Rafique S, et al. (2017) Therapeutic Strategies of Clustered Regularly Interspaced Palindromic Repeats-Cas Systems for Different Viral Infections. Viral Immunol 30: 552-559.
100. Roesch F, OhAinle M, Emerman M (2018) A CRISPR screen for factors regulating SAMHD1 degradation identifies IFITMs as potent inhibitors of lentiviral particle delivery. Retrovirology 15: 26.

101. Wu HY, Cao CY (2018) The application of CRISPR-Cas9 genome editing tool in cancer immunotherapy. Brief Funct Genomics.

102. Wang C, Jin H, Gao D, Wang L, Evers B, et al. (2018) A CRISPR screen identifies CDK7 as a therapeutic target in hepatocellular carcinoma. Cell Res.

103. Wang G, Chow RD, Ye L, Guzman CD, Dai X, et al. (2018) Mapping a functional cancer genome atlas of tumor suppressors in mouse liver using AAV-CRISPRmediated direct in vivo screening. $S c i$ Adv 4: eaao5508.

104. Chira S, Gulei D, Hajitou A, Berindan-Neagoe I (2018) Restoring the p53 'Guardian' Phenotype in p53-Deficient Tumor Cells with CRISPR/Cas9. Trends Biotechnol 18: 30048-30049.

105. Norouzi-Barough L, Sarookhani M, Salehi R, Sharifi M, Moghbelinejad S (2018) CRISPR/Cas9, a new approach to successful knockdown of ABCB1/P-glycoprotein and reversal of chemosensitivity in human epithelial ovarian cancer cell line. Iran J Basic Med Sci 21: 181-187.

106. Cradick TJ, Fine EJ, Antico CJ, Bao G (2013) CRISPR/Cas9 systems targeting $\hat{I}^{2}$ globin and CCR5 genes have substantial off-target activity. Nucleic Acids Res 41: 9584-9592. [Crossref]

107. Sakurai T, Watanabe S, Kamiyoshi A, Sato M, Shindo T (2014) A single blastocys assay optimized for detecting CRISPR/Cas9 system-induced indel mutations in mice. BMC Biotechnol 14: 69.

108. Ding Q, Strong A, Patel KM, Ng SL, Gosis BS, et al. (2014) Permanent alteration of PCSK9 with in vivo CRISPR-Cas9 genome editing. Circ Res 115: 488-492. [Crossref]

109. Nakamura K, Fujii W, Tsuboi M, Tanihata J, Teramoto N, et al. (2014) Generation of muscular dystrophy model rats with a CRISPR/Cas system. Sci Rep 4: 5635. [Crossref]

110. Horii T, Arai Y, Yamazaki M, Morita S, Kimura M, et al. (2014) Validation of microinjection methods for generating knockout mice by CRISPR/Cas-mediated genome engineering. Sci Rep 4: 4513 .

Copyright: $\odot 2018$ Adamov N. This is an open-access article distributed under the terms of the Creative Commons Attribution License, which permits unrestricted use, distribution, and reproduction in any medium, provided the original author and source are credited. 